\title{
Angioplastia Primária no Infarto Agudo do Miocárdio: Existe Diferença de Resultados entre as Angioplastias Realizadas Dentro e Fora do Horário de Rotina?
}

\author{
Cristiano de Oliveira Cardoso ${ }^{1}$, Alexandre Schaan de Quadros ${ }^{1}$, Ismael Voltolini ${ }^{1}$, \\ Alexandre Damiani Azmus ${ }^{1}$, Carlos Roberto Cardoso', Juliana Sebben' ${ }^{1}$, Dulce Welter ${ }^{1}$, \\ Anibal Pereira Abelin', Marciane Rover', Felipe Baldissera', Márcio Bosco ${ }^{1}$, Délcio Rodrigues',

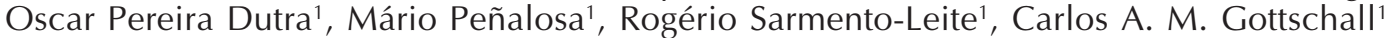

\section{RESUMO}

Introdução: Estudos demonstram que as angioplastias primárias realizadas fora do horário de rotina estão relacionadas a pior prognóstico. É objetivo deste estudo avaliar os desfechos das angioplastias primárias realizadas dentro e fora do horário de rotina do serviço de hemodinâmica. Método: Estudo de coorte prospectivo, incluindo 112 pacientes consecutivamente atendidos por infarto agudo do miocárdio com supradesnivelamento do segmento ST (IAM) entre dezembro de 2009 e janeiro de 2010. Características clínicas e angiográficas e evolução hospitalar foram registradas em banco de dados específico. Houve dois grupos para comparação: grupo A, IAM tratado entre as 20 horas e as 8 horas e B, IAM tratado entre as 8 horas e as 20 horas. Resultados: A amostra incluiu 44 pacientes no grupo A e 68 no grupo B. As características basais foram semelhantes em ambos os grupos. O tempo porta-balão foi significativamente maior no grupo A (133 minutos vs. 90 minutos; $P<0,001)$. No entanto, não houve diferença significante entre os grupos A e B em relação a mortalidade (13,7\% vs. $5,9 \% ; P=0,28)$, reinfarto $(6,8 \%$ vs. $4,4 \% ; P=0,90)$, evolução para choque cardiogênico $(11,4 \%$ vs. $4,4 \%$; $P=0,30)$, trombose de stent $(6,8 \%$ vs. $1,5 \% ; \mathrm{P}=0,33)$ ou presença de sangramento maior $(2,3 \%$ vs. $1,5 \%$; P > 0,99). O único preditor do desfecho hospitalar combinado foi a frequência cardíaca à admissão (razão de chance 1,02; P $<0,001$ ). Conclusão: Pacientes com IAM apresentam taxas de desfechos clínicos semelhantes, independentemente do horário de realização da angioplastia, porém o tempo porta-balão é significativamente maior nos pacientes tratados entre as 20 horas e as 8 horas.

DESCRITORES: Infarto do miocárdio. Angioplastia. Reperfusão miocárdica.

\section{ABSTRACT}

Primary Percutaneous Coronary Intervention in Acute Myocardial Infarction: Is There a Difference in the Outcomes of Normal and Off-hours Procedures?

Background: Previous studies have reported that off-hours primary percutaneous coronary intervention is related to worse prognosis. The objective of this study is to evaluate the outcomes of normal and off-hours primary percutaneous coronary interventions. Method: A prospective observational study including 112 consecutive patients with ST elevation myocardial infarction (MI) was conducted from December 2009 to January 2010. Clinical and angiographic characteristics and in-hospital follow up were registered in a specific database. There were two groups for comparison: group A, Ml treated between $8 \mathrm{pm}$ and $8 \mathrm{am}$ and group B, Ml treated between 8 am and $8 \mathrm{pm}$. Results: The sample included 44 patients in group A and 68 in group B. Baseline characteristics were similar in both groups. Door-to-balloon time was significantly longer in group A (133 minutes vs. 90 minutes; $\mathrm{P}<0.001)$. However, there was no significant difference between groups $\mathrm{A}$ and $\mathrm{B}$ regarding mortality $(13.7 \%$ vs. $5.9 \%$; $P=0.28)$, reinfarction $(6.8 \%$ vs. $4.4 \%$; $P=0.90)$, progression to cardiogenic shock $(11.4 \%$ vs. $4.4 \% ; \mathrm{P}=0.30)$, stent thrombosis $(6.8 \%$ vs. $1.5 \% ; \mathrm{P}=0.33)$, or major bleeding $(2.3 \%$ vs. $1.5 \%$; $\mathrm{P}>0.99)$. The only predictor of combined in-hospital outcome was heart rate at admission (odds ratio 1.02; $\mathrm{P}<0.001$ ). Conclusion: Patients with $\mathrm{MI}$ have similar in-hospital clinical outcomes during normal and off-hours primary percutaneous coronary intervention. However, door-to-balloon time is significantly longer during off-hours procedures.

KEY-WORDS: Myocardial infarction. Angioplasty. Myocardial reperfusion.

${ }^{1}$ Instituto de Cardiologia do Rio Grande do Sul - Fundação Universitária de Cardiologia (IC-FUC) - Porto Alegre, RS, Brasil.

Correspondência: Cristiano de Oliveira Cardoso. Av. Princesa Isabel,

395 - Santana - Porto Alegre, RS, Brasil - CEP 90620-000

E-mail: cro_cardoso@yahoo.com.br

Recebido em: 4/7/2010 • Aceito em: 6/9/2010 
A terapêutica de reperfusão para infarto agudo do miocárdio com supradesnivelamento do segmento ST, seja por intervenção coronária percutânea primária ou trombólise por via endovenosa, comprovadamente reduz a mortalidade cardiovascular. ${ }^{1-3} \mathrm{~A}$ intervenção coronária percutânea primária mostrou-se superior à terapia trombolítica, pois é capaz de restaurar o fluxo coronário em mais de $90 \%$ dos casos, reduzir a taxa de reinfarto, aumentar a sobrevida e apresentar menores taxas de acidente vascular encefálico. ${ }^{4-9}$ Diante desses benefícios, as diretrizes atuais recomendam que pacientes com infarto agudo do miocárdio com supradesnivelamento do segmento ST e início de apresentação dos sintomas inferior a 12 horas têm indicação classe I de tratamento por intervenção coronária percutânea primária em centros especializados e com capacidade de realização desse procedimento dentro dos primeiros 90 minutos. $^{7,10,11}$

A conduta para realização dos procedimentos durante o dia e a noite varia muito na maior parte dos hospitais. Habitualmente, a maioria dos centros não possui um plantão presencial noturno, o que poderia tornar o tempo porta-balão mais prolongado nesse período. Estudos sugerem que o tempo porta-balão e a mortalidade podem ser elevados no período noturno comparativamente ao horário diurno. ${ }^{12-14}$

Dados a respeito desse assunto, no entanto, têm sido pouco relatados na literatura nacional. Por conseguinte, o objetivo deste trabalho é avaliar os desfechos das intervenções coronárias percutâneas primárias em horário de rotina e de plantão em um centro cardiológico de referência.

\section{MÉTODO}

\section{Delineamento}

Estudo observacional com coleta de dados prospectiva.

\section{Amostra}

Pacientes com diagnóstico de infarto agudo do miocárdio com supradesnivelamento do segmento ST e submetidos a intervenção coronária percutânea primária foram prospectivamente alocados para o estudo. Os critérios de inclusão foram definidos como diagnóstico clínico e eletrocardiográfico de infarto agudo do miocárdio com indicação de tratamento por intervenção coronária percutânea primária. Foram excluídos pacientes que apresentassem infarto agudo do miocárdio com mais de 12 horas de evolução (exceto aqueles em choque cardiogênico), menores de 18 anos ou recusa a participar do estudo. Os pacientes foram acompanhados no período hospitalar, sendo divididos em dois grupos para comparação: grupo A, infarto agudo do miocárdio entre as 20 horas e as 8 horas e grupo B, infarto agudo do miocárdio entre as 8 horas e as 20 horas. Todos os pacientes foram entrevistados na admissão hospitalar e acompanhados durante a internação por um dos investigadores do estudo. Todos os pacientes assinaram termo de consentimento e o protocolo foi aprovado pelo comitê de ética e pesquisa local.

\section{Características institucionais}

O Instituto de Cardiologia do Rio Grande do Sul Fundação Universitária de Cardiologia (IC-FUC) possui um grande volume de pacientes em tratamento para cardiopatia isquêmica. Realiza cerca de 10 mil procedimentos percutâneos diagnósticos e terapêuticos por ano, sendo a frequência de intervenção coronária percutânea primária aproximadamente de uma a duas vezes por dia. O hospital disponibiliza o serviço de intervenção coronária percutânea primária durante 24 horas por dia nos sete dias da semana. O laboratório de hemodinâmica é localizado no andar térreo, no mesmo nível do setor de emergência, com cerca de 100 metros de distância entre os dois setores, o que dispensa a necessidade de uso de elevador. O serviço de hemodinâmica possui 14 operadores, todos com experiência em procedimentos de intervenção coronária percutânea primária, conforme as recomendações das diretrizes. ${ }^{7,10}$

\section{Procedimento de angioplastia primária}

Os pacientes, após diagnóstico de infarto agudo do miocárdio, recebiam, na emergência, dose de ataque de 300 mg a 500 mg de ácido acetilsalicílico e $300 \mathrm{mg}$ a $600 \mathrm{mg}$ de clopidogrel. O uso de morfina, heparina endovenosa, nitrato sublingual/endovenoso ou betabloqueador ficava a critério do médico plantonista. Assim que possível, o enfermo era conduzido ao setor de hemodinâmica para a realização do procedimento.

Uma vez no laboratório, o paciente era submetido a cinecoronariografia e, após confirmação diagnóstica de infarto agudo do miocárdio, a heparinização plena em bolus na dose de $60 \mathrm{U} / \mathrm{kg}$ a $100 \mathrm{U} / \mathrm{kg}$. O procedimento de intervenção coronária percutânea primária foi realizado de acordo com as normas atuais, sendo a via de acesso definida pelo médico hemodinamicista. Pré-dilatação, administração de inibidores de glicoproteína IIb/IIla, tromboaspiração e implante de stent foram utilizados de acordo com o julgamento do operador. A instalação de balão intra-aórtico ficou restrita aos pacientes com choque cardiogênico.

\section{Definições}

Infarto agudo do miocárdio foi definido como: (1) presença de dor em repouso associada a supradesnivelamento do segmento ST de $1 \mathrm{~mm}$ ou em pelo menos duas derivações contíguas; e (2) presença de dor em pacientes com bloqueio de ramo esquerdo presumível novo. Seguindo a definição universal de infarto agudo ${ }^{15}$, o tipo de infarto agudo do miocárdio foi classificado em: tipo 1, infarto agudo do miocárdio espontâneo relacionado a evento coronário primário; tipo 2, 
Cardoso CO, et al. Angioplastia Primária no Infarto Agudo do Miocárdio: Existe Diferença de Resultados entre as Angioplastias Realizadas Dentro e Fora do Horário de Rotina? Rev Bras Cardiol Invasiva. 2010;18(3):273-80.

infarto agudo do miocárdio secundário a isquemia por aumento da demanda ou redução da oferta de oxigênio; tipo 3, morte cardíaca; tipo 4a, infarto agudo do miocárdio relacionado a intervenção percutânea; tipo 4b, infarto agudo do miocárdio por trombose de stent documentada à angiografia; e tipo 5, infarto agudo do miocárdio relacionado a cirurgia de revascularização miocárdica.

Tempo porta-balão foi determinado como o tempo compreendido entre a admissão hospitalar na emergência e a primeira insuflação do balão de angioplastia ou liberação do stent (nos casos de stent direto). O tempo decorrido entre o início dos sintomas e a admissão hospitalar foi definido como delta $T(\Delta t)$.

Trombose aguda de stent foi caracterizada como oclusão súbita do vaso tratado até 24 horas após o procedimento, confirmada por angiografia. Trombose subaguda foi definida como trombose do stent no período de 24 horas a 30 dias após o implante.

Sangramento menor foi definido na presença de hemorragia clinicamente evidente com queda de hemoglobina entre $3 \mathrm{~g} / \mathrm{dl}$ e $5 \mathrm{~g} / \mathrm{dl}$ ou hematócrito entre $9 \%$ e $15 \%$. Sangramento maior foi definido quando da ocorrência de hemorragia clinicamente evidente com queda dos níveis de hemoglobina $>5 \mathrm{~g} / \mathrm{dl}$ ou hematócrito $>15 \%$ ou desenvolvimento de acidente vascular encefálico hemorrágico.

Reinfarto foi definido como nova elevação enzimática após primeiro descenso da curva natural das enzimas marcadoras de necrose, acompanhada de nova alteração eletrocardiográfica com supradesnivelamento do segmento ST.

\section{Desfechos intra-hospitalares}

Os desfechos intra-hospitalares utilizados para comparação entre os grupos foram: mortalidade intra-hospitalar, reinfarto, acidente vascular encefálico, sangramento maior ou menor, trombose de stent ou insuficiência renal com necessidade de diálise.

\section{Análise estatística}

Os dados foram prospectivamente armazenados em um banco de dados específicos no programa ACCESS. Para análise, utilizou-se o programa estatístico SPSS versão 11.0 para Windows. As variáveis categóricas foram descritas como números absolutos e porcentuais e comparadas com o teste de qui-quadrado. As variáveis contínuas foram apresentadas como média e desvio padrão e comparadas com o teste $t$. O teste de MannWhitney foi utilizado em casos de variáveis com distribuição não normal. Regressão logística múltipla foi aplicada para identificar possíveis preditores de complicações intra-hospitalares. Foram utilizadas, nessa análise, variáveis com significância estatística na análise univariada ou sem significância estatística, mas com relevância clinica. $\mathrm{O}$ modelo de regressão utilizado foi o de Poisson. Foi considerada significância estatística valor de $\mathrm{P}$ bicaudal $<0,05$.

\section{RESULTADOS}

Entre dezembro de 2009 e janeiro de 2010, 115 pacientes foram tratados com intervenção coronária percutânea primária pelo diagnóstico de infarto agudo do miocárdio, sendo 3 casos excluídos da análise por preenchimento incompleto do protocolo. No total, 112 pacientes compuseram a amostra, sendo 44 pacientes incluídos no grupo A (intervenção coronária percutânea primária das 20 horas às 8 horas) e 68 no grupo B (intervenção coronária percutânea primária das 8 horas às 20 horas).

Não existiu diferença estatística $(P=0,93)$ em relação ao tipo de infarto agudo do miocárdio conforme a classificação universal de infarto nos grupos A e B: tipo $1(93,2 \%$ vs. $91,2 \%)$, tipo $2(2,3 \%$ vs. $2,9 \%)$, tipo 4 a (0 vs. $1,5 \%)$, tipo 4 b $(2,3 \%$ vs. $2,9 \%)$ e tipo $5(2,3 \%$ e 1,5\%). Casos de intervenção coronária percutânea de resgate ocorreram em 2,3\% no grupo A e em 2,9\% no grupo $B(P=0,92)$.

Na Tabela 1 são apresentados os resultados dos dois grupos. Observa-se que ambos os grupos apresentavam tanto características clínicas quanto achados eletrocardiográficos muito semelhantes. O grupo de pacientes submetidos a angioplastia primária no período noturno apresentou tempo menor entre o início dos sintomas e o atendimento na emergência $(\Delta \mathrm{t}$ de $4,8 \pm$ 4,1 horas no grupo A vs. 5,7 $\pm 5,3$ horas no grupo B; $P=0,31)$, mas tempo porta-balão significativamente maior que aqueles tratados durante o dia $(133 \pm 102$ minutos no grupo A vs. $90 \pm 120$ minutos no grupo $B$; $P<0,001)$. Considerando que houve grande variabilidade no tempo porta-balão, a Figura 1 demonstra suas distribuições por percentil. Desse modo, observa-se que independentemente do turno o tempo-porta balão é menor ou igual a 90 minutos em $50 \%$ dos casos.

As características angiográficas e técnicas dos procedimentos são detalhadas na Tabela 2. Não existiu diferença estatisticamente significante entre os grupos de comparação no que tange a via de acesso, comprometimento vascular, vaso culpado e detalhes técnicos dos procedimentos. Tromboaspiração com cateter específico foi utilizada em 15,9\% dos pacientes do grupo A e em $27,9 \%$ dos pacientes do grupo $B(P=0,3)$.

Com relação à terapia antiplaquetária, verificou-se que a dose de ataque de clopidogrel foi semelhante em ambos os grupos, variando entre $300 \mathrm{mg}$ (40,9\% vs. $41,2 \% ; \mathrm{P}=0,8)$ e $600 \mathrm{mg}(59,1 \%$ vs. 58,8\%; $\mathrm{P}=0,79)$. Inibidor de glicoproteína IIb/IIla foi utilizado em 29,5\% dos pacientes do grupo A e em $25 \%$ dos pacientes do grupo $B(P=0,6)$.

Uso de balão de contrapulsação aórtica foi semeIhante em ambos os grupos (2,3\% vs. $1,5 \%$; $P=0,75)$; 
TABELA 1

Características clínicas e eletrocardiográficas dos pacientes com infarto agudo do miocárdio submetidos a angioplastia primária

\begin{tabular}{|c|c|c|c|}
\hline Variável & $\begin{array}{c}\text { Grupo A } \\
(n=44)\end{array}$ & $\begin{array}{l}\text { Grupo B } \\
(\mathbf{n}=68)\end{array}$ & $\mathbf{P}$ \\
\hline \multicolumn{4}{|l|}{ Características clínicas } \\
\hline Idade, anos & $58,9 \pm 10,5$ & $58,6 \pm 10,7$ & 0,85 \\
\hline Peso, kg & $75,5 \pm 14,8$ & $75 \pm 16,1$ & 0,83 \\
\hline Altura, $\mathrm{cm}$ & $166 \pm 8,8$ & $168,8 \pm 9,3$ & 0,81 \\
\hline Sexo masculino, \% & 58,8 & 67,6 & 0,37 \\
\hline Tabagismo ativo, \% & 52,3 & 42,6 & 0,57 \\
\hline Ex-tabagista, \% & 27,3 & 35,3 & 0,57 \\
\hline Hipertensão arterial, \% & 75 & 58,8 & 0,08 \\
\hline Diabetes, \% & 20,5 & 16,2 & 0,74 \\
\hline Uso de insulina, \% & 4,5 & 1,5 & 0,56 \\
\hline Dislipidemia, \% & 47,7 & 27,9 & 0,53 \\
\hline História familiar, \% & 38,6 & 38,2 & $>0,99$ \\
\hline ICP prévia, \% & 15,9 & 13,2 & 0,67 \\
\hline CRM prévia, \% & 2,3 & 0 & 0,33 \\
\hline IAM prévio, \% & 34,1 & 22,1 & 0,34 \\
\hline Frequência cardíaca, bpm & $74,6 \pm 18,5$ & $77,4 \pm 19,2$ & 0,45 \\
\hline Pressão arterial sistólica, mmHg & $128,8 \pm 30,8$ & $137,2 \pm 30,5$ & 0,56 \\
\hline Pressão arterial diastólica, mmHg & $80,68 \pm 18,5$ & $82,3 \pm 18,3$ & 0,64 \\
\hline Killip & & & 0,96 \\
\hline 1 & 81 & 83,8 & \\
\hline ॥ & 13,6 & 11,8 & \\
\hline III & 0 & 0 & \\
\hline IV & 4,5 & 4,4 & \\
\hline \multicolumn{4}{|l|}{ Achados eletrocardiográficos, \% } \\
\hline IAM anterior & 36,4 & 33,8 & 0,78 \\
\hline IAM de ventrículo direito & 6,8 & 8,8 & 0,98 \\
\hline BAVT & 2,3 & 1,5 & $>0,99$ \\
\hline
\end{tabular}

BAVT = bloqueio atrioventricular total; CRM = cirurgia de revascularição miocárdica; IAM = infarto agudo do miocárdio; ICP = intervenção coronária percutânea; $\mathrm{n}$ = número de pacientes.

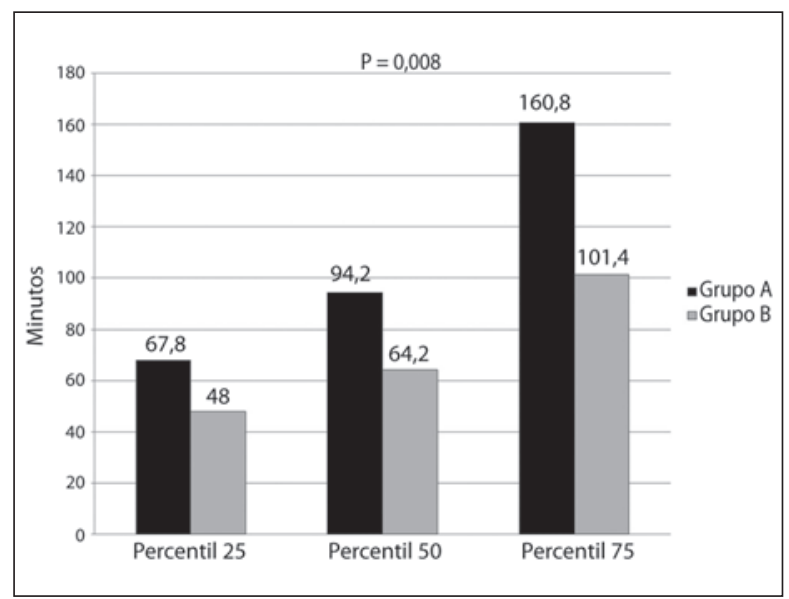

Figura 1 - Distribuição do tempo porta-balão por percentil entre os grupos de comparação. no entanto, maior porcentual de pacientes do grupo A evoluiu para choque cardiogênico $(11,4 \%$ vs. 4,4\%; $\mathrm{P}=0,3)$. A necessidade de implante de marca-passo temporário foi significativamente maior no grupo $A$ $(9,1 \%$ vs. $1,5 \% ; P=0,005)$.

Quanto aos desfechos intra-hospitalares observados, não houve diferença estatisticamente significante entre os pacientes submetidos a intervenção coronária percutânea primária nos períodos noturno e diurno em relação a mortalidade $(13,7 \%$ vs. 5,9\%; $P=0,28)$, reinfarto $(6,8 \%$ vs. $4,4 \% ; \mathrm{P}=0,9)$, sangramento maior $(2,3 \%$ vs. $1,5 \% ; P>0,99)$ e trombose de stent $(6,8 \%$ vs. $1,5 \% ; P=0,29)$. Necessidade de diálise $(2,3 \%$ vs. $7,4 \% ; P=0,05)$ e taxa de sangramento menor (0 vs. $7,4 \% ; P=0,04)$ foram significativamente mais elevadas no grupo B. Acidente vascular encefálico não ocorreu em ambos os grupos. 
TABELA 2

Características angiográficas e técnicas dos procedimentos de angioplastia primária

\begin{tabular}{|c|c|c|c|}
\hline Variável & $\begin{array}{c}\text { Grupo A } \\
(n=44)\end{array}$ & $\begin{array}{l}\text { Grupo B } \\
(n=68)\end{array}$ & $\mathbf{P}$ \\
\hline Via de acesso, $\%$ & & & 0,19 \\
\hline Femoral & 90,9 & 79,4 & \\
\hline Radial & 9,1 & 20,6 & \\
\hline Comprometimento vascular, \% & & & 0,59 \\
\hline Uniarterial & 43,9 & 50 & \\
\hline Biarterial & 34,1 & 25 & \\
\hline Triarterial & 22 & 25 & \\
\hline Lesão de tronco de coronária esquerda, \% & 4,5 & 0 & 0,07 \\
\hline Vaso culpado, \% & & & 0,62 \\
\hline DA ou diagonal & 31,9 & 32,3 & \\
\hline CX ou marginal & 6,8 & 8,9 & \\
\hline CD ou ramos & 61,3 & 58,8 & \\
\hline Ramos envolvidos, \% & 27,3 & 29,4 & 0,84 \\
\hline Trombo associado, \% & 61,4 & 75 & 0,93 \\
\hline Fluxo TIMI pré-procedimento, \% & & & 0,92 \\
\hline 0 e 1 & 73,1 & 70,8 & \\
\hline 2 e 3 & 26,9 & 29,2 & \\
\hline Fluxo TIMI pós-procedimento, \% & & & 0,6 \\
\hline 0 e 1 & 4,9 & 6,3 & \\
\hline 2 e 3 & 95,1 & 93,7 & \\
\hline Pré-dilatação, \% & 61,4 & 61,8 & 0,96 \\
\hline Pós-dilatação, \% & 27,3 & 16,2 & 0,23 \\
\hline Uso de stent, \% & 93,1 & 91,2 & 0,56 \\
\hline Diâmetro do stent, mm & $3,2 \pm 0,5$ & $3,1 \pm 0,45$ & 0,59 \\
\hline Comprimento do stent, $\mathrm{mm}$ & $17,8 \pm 4,7$ & $18,2 \pm 5,3$ & 0,71 \\
\hline Pressão de liberação do stent, atm & $14,4 \pm 2,9$ & $14,5 \pm 2,8$ & 0,83 \\
\hline
\end{tabular}

Na Tabela 3 são demonstrados os preditores clínicos e angiográficos relacionados a desfechos intra-hospitalares combinados. O único preditor com significância estatística foi a frequência cardíaca > 100 bpm no momento da admissão hospitalar.

\section{DISCUSSÃO}

Neste estudo, demonstramos os resultados da angioplastia primária em um centro cardiológico de referência no Sul do Brasil, confirmando sua aplicabilidade e efetividade quando realizada durante as 24 horas do dia na prática clínica do mundo real. Como é sabido, a intervenção coronária percutânea primária tem indicação classe I em pacientes com infarto agudo do miocárdio com supradesnivelamento do segmento ST, pois é possível restaurar o fluxo coronário em mais de $90 \%$ dos casos e diminuir os índices de mortalidade, reinfarto e acidente vascular encefálico..$^{5-7}$ Por isso, torna-se importante uma estratégia de saúde que dis- ponibilize o serviço nas 24 horas do dia, uma vez que cerca de $50 \%$ dos casos de infarto agudo ocorrem fora do horário regular de trabalho. ${ }^{16}$

Estudos prévios sugerem que as intervenções coronárias percutâneas primárias realizadas fora do horário de rotina estão associadas a aumento da mortalidade. ${ }^{12,13,17}$ Embora sem uma explicação precisa, acredita-se que a maior mortalidade decorra do maior tempo de chegada à emergência que os infartos atendidos durante o dia. Em nosso estudo, no entanto, os pacientes com infarto agudo do miocárdio tratados no horário noturno apresentaram-se na emergência cerca de uma hora mais cedo que os demais pacientes. Logo, o teórico retardo na apresentação do infarto não foi registrado em nossa casuística. Além disso, o perfil tanto clínico como hemodinâmico foi similar entre os dois grupos, o que impede que a maior mortalidade observada em nosso estudo, mesmo que sem diferença estatística, seja explicada unicamente pelo $\Delta$ t. 
TABELA 3

Análise multivariada para os preditores clínicos e angiográficos de desfecho hospitalar

\begin{tabular}{lccc} 
Variável & Razão de chance & IC & P \\
\hline Frequência cardíaca > 100 bpm & 1,02 & $1,01-1,03$ & $<0,001$ \\
Pressão arterial sistólica, mmHg & 0,99 & $0,98-1$ & 0,62 \\
Pressão arterial diastólica, mmHg & 0,97 & $0,95-1$ & 0,051 \\
Killip II & 1,79 & $0,8-2,59$ & 0,15 \\
Killip IV & 1,9 & $0,78-4,62$ & 0,19 \\
ICP primária entre as 20 horas e as 8 horas & 1,04 & $0,4-2,59$ & 0,92 \\
Lesão de bifurcação & 1,64 & $0,58-4,6$ & 0,4 \\
\hline
\end{tabular}

IC = intervalo de confiança; ICP = intervenção coronária percutânea.

O tempo entre o diagnóstico de infarto e o início da reperfusão exerce papel fundamental nos desfechos clínicos e hospitalares. ${ }^{18}$ Por isso, as diretrizes atuais são categóricas em recomendar que os tempos portabalão e porta-agulha sejam inferiores a 90 minutos e a 30 minutos, respectivamente. ${ }^{7,10,11}$ Em grande estudo envolvendo mais de 60 mil pacientes com infarto agudo do miocárdio ${ }^{19}$, verificou-se que a administração de terapia trombolítica por via venosa sofre pouca interferência em relação ao horário do infarto. No entanto, o tempo porta-balão foi significativamente maior nos pacientes tratados com intervenção coronária percutânea primária durante a noite. Nosso estudo demonstrou que o tempo porta-balão variou amplamente na amostra estudada, principalmente nas intervenções coronárias percutâneas realizadas fora do turno regular de trabaIho. Uma possível explicação seria o menor contingente de pessoas trabalhando nos hospitais durante a noite. Como é sabido, os turnos de trabalho noturnos são em esquema de plantão e, portanto, com menor número de funcionários, o que pode acarretar retardo no tempo porta-balão e contribuir com maior impacto nos desfechos hospitalares.

Os antiagregantes plaquetários possuem papel importante no tratamento farmacológico das síndromes coronárias agudas. Ácido acetilsalicílico, clopidogrel e inibidores de glicoproteína IIb/IIla são indicados como terapia adjunta na reperfusão miocárdica com níveis variados de recomendação. 7,10,11 Nosso estudo demonstrou que a utilização de clopidogrel na dose de $600 \mathrm{mg}$ chegou a quase $60 \%$ dos pacientes. No entanto, o uso de inibidor de glicoproteína IIb/Illa é muito inferior se comparado aos registros prévios. ${ }^{16,17,20}$

Embora a taxa de desfechos hospitalares não tenha apresentado diferença estatística, fica claro que a prevalência de casos de trombose de stent, mortalidade, reinfarto e sangramento maior foi nitidamente mais elevada no grupo de angioplastia no horário do plantão. Em recente estudo observacional, foi demonstrado pelo National Cardiovascular Data Registry (NCDR) ${ }^{19}$ que a mortalidade cardiovascular foi reduzida nos últimos anos entre os pacientes com infarto agudo do miocárdio com supradesnivelamento do segmento ST para cerca de 5\%. Em nosso estudo a mortalidade diurna é compatível com esse registro, porém a mortalidade noturna é praticamente o dobro. A explicação para o fenômeno observado não é clara aos autores, pois o perfil clínico dos pacientes e as características técnicas dos procedimentos foram muito semelhantes. Certamente o retardo no tempo porta-balão deve ter desempenhado papel importante nos desfechos clínicos observados. ${ }^{21}$

\section{Limitações do estudo}

O presente estudo tem limitações que devem ser consideradas: análise em centro único e com amostra pequena de pacientes. Os desfechos restringem-se apenas à fase intra-hospitalar, o que limita qualquer conclusão com respeito à evolução tardia dos pacientes tratados no horário regular e no esquema de plantão. Nossos resultados poderão ser diferentes dos resultados de estudos que vierem a ser realizados com maior tamanho de amostra, uma vez que este estudo não possui poder adequado para avaliação de desfechos clínicos.

\section{Perspectivas futuras}

A terapêutica de reperfusão no infarto agudo do miocárdio exerce papel fundamental na redução de mortalidade e desfechos cardiovasculares. ${ }^{22}$ Conforme demonstrado neste estudo, o procedimento de angioplastia primária é factível nas 24 horas do dia e durante os sete dias da semana. É imperativo que as políticas de saúde incorporem os procedimentos de intervenção coronária percutânea primária nas estratégias de saúde pública.

Mesmo em centro de referência e com larga experiência em intervenção coronária percutânea primária, fica demonstrado que a taxa de desfechos pode ser diferente dependendo do turno de trabalho. Por isso, torna-se necessária análise institucional focada no fluxo 
Cardoso CO, et al. Angioplastia Primária no Infarto Agudo do Miocárdio: Existe Diferença de Resultados entre as Angioplastias Realizadas Dentro e Fora do Horário de Rotina? Rev Bras Cardiol Invasiva. 2010;18(3):273-80.

de entrada e no encaminhamento dos pacientes, com o intuito de minimizar o tempo porta-balão e de dar toda a assistência aos pacientes infartados. ${ }^{23}$

\section{CONCLUSÃO}

Pacientes com infarto agudo do miocárdio apresentam taxas de desfechos clínicos semelhantes, independentemente do horário de realização da angioplastia primária. No entanto, o tempo porta-balão é significativamente maior nos pacientes tratados entre as 20 horas e as 8 horas.

\section{AGRADECIMENTO}

Agradecemos especialmente aos colegas hemodinamicistas Cláudio A. Ramos de Moraes, Cláudio Vasques de Moraes, Henrique Basso Gomes, Júlio Vinícius Teixeira, Flávio Celso Leboute, La Hore Corrêa Rodrigues, Luis Maria Yordi e Mauro Régis Moura, que, embora não tenham preenchido critérios de autoria, foram fundamentais na realização dos procedimentos de angioplastia primária.

\section{CONFLITO DE INTERESSES}

Os autores declararam inexistência de conflito de interesses relacionado a este manuscrito.

\section{REFERÊNCIAS}

1. Gruppo Italiano per lo Studio della Streptochinasi nell'Infarto Miocardico (GISSI). Effectiveness of intravenous thrombolytic treatment in acute myocardial infarction. Lancet. 1986;1(8478): 397-402.

2. Second International Study of Infarct Survival Collaborative Group. Randomised trial of intravenous streptokinase, oral aspirin, both, or neither among 17,187 cases of suspected acute myocardial infarction: ISIS-2. Lancet. 1988;2(8607):349-60.

3. O'Neill W, Timmis GC, Bourdillon PD, Lai P, Ganghadarhan $\mathrm{V}$, Walton J Jr, et al. A prospective randomized clinical trial of intracoronary streptokinase versus coronary angioplasty for acute myocardial infarction. N Engl J Med. 1986;314(13):812-8.

4. Andersen HR, Nielsen TT, Rasmussen K, Thuesen L, Kelbaek $\mathrm{H}$, Thayssen $\mathrm{P}$, et al. A comparison of coronary angioplasty with fibrinolytic therapy in acute myocardial infarction. $\mathrm{N}$ Engl J Med. 2003;349(8):733-42.

5. Grines C, Patel A, Zijlstra F, Weaver WD, Granger C, Simes RJ. Primary coronary angioplasty compared with intravenous thrombolytic therapy for acute myocardial infarction: sixmonth follow up and analysis of individual patient data from randomized trials. Am Heart J. 2003;145(1):47-57.

6. Keeley EC, Boura JA, Grines CL. Primary angioplasty versus intravenous thrombolytic therapy for acute myocardial infarction: a quantitative review of 23 randomised trials. Lancet. 2003;361(9351):13-20.

7. Mattos LA, Lemos Neto PA, Rassi Junior A, Marin-Neto JA, Souza AGMR, Devito FS. Diretrizes da Sociedade Brasileira de Cardiologia: intervenção coronária percutânea e métodos adjuntos diagnósticos em cardiologia intervencionista (II Edição 2008). Rev Bras Cardiol Invasiva. 2008;16(2 Supl 2):9-88.

8. Nielsen PH, Maeng $M$, Busk $M$, Mortensen LS, Kristensen SD, Nielsen TT, et al. Primary angioplasty versus fibrinolysis in acute myocardial infarction: long-term follow-up in the
Danish acute myocardial infarction 2 trial. Circulation. 2010;121(13):1484-91.

9. Zijlstra F, de Boer MJ, Hoorntje JC, Reiffers S, Reiber JH, Suryapranata H. A comparison of immediate coronary angioplasty with intravenous streptokinase in acute myocardial infarction. N Engl J Med. 1993;328(10):680-4.

10. Antman EM, Anbe DT, Armstrong PW, Bates ER, Green LA, Hand $M$, et al. ACC/AHA guidelines for the management of patients with ST-elevation myocardial infarction-executive summary: a report of the American College of Cardiology/ American Heart Association Task Force on Practice Guidelines (Writing Committee to Revise the 1999 Guidelines for the Management of Patients With Acute Myocardial Infarction). Circulation. 2004;110(5):588-636.

11. Kushner FG, Hand M, Smith SC Jr, King SB $3^{\text {rd }}$, Anderson JL, Antman EM, et al. 2009 Focused Updates: ACC/AHA Guidelines for the Management of Patients With ST-Elevation Myocardial Infarction (updating the 2004 Guideline and 2007 Focused Update) and ACC/AHA/SCAI Guidelines on Percutaneous Coronary Intervention (updating the 2005 Guideline and 2007 Focused Update): a report of the American College of Cardiology Foundation/American Heart Association Task Force on Practice Guidelines. Circulation. 2009;120(22):2271-306.

12. De Luca G, Suryapranata H, Ottervanger JP, van't Hof AW, Hoorntje JC, Gosselink AT, et al. Circadian variation in myocardial perfusion and mortality in patients with ST-segment elevation myocardial infarction treated by primary angioplasty. Am Heart J. 2005;150(6):1185-9.

13. Henriques JP, Haasdijk AP, Zijlstra F. Outcome of primary angioplasty for acute myocardial infarction during routine duty hours versus during off-hours. J Am Coll Cardiol. 2003;41(12):2138-42.

14. Saleem MA, Kannam H, Aronow WS, Weiss MB, Kalapatapu $\mathrm{K}$, Pucillo AL, et al. The effects of off-normal hours, age, and gender for coronary angioplasty on hospital mortality in patients undergoing coronary angioplasty for acute myocardial infarction. J Am Coll Cardiol. 2004;93(6):763-4.

15. Thygesen K, Alpert JS, White HD, Jaffe AS, Apple FS, Galvani M, et al. Universal definition of myocardial infarction. Circulation. 2007;116(22):2634-53.

16. Uyarel H, Ergelen M, Akkaya E, Ayhan E, Demirci D, Gul $M$, et al. Impact of day versus night as intervention time on the outcomes of primary angioplasty for acute myocardial infarction. Catheter Cardiovasc Interv. 2009;74(6):826-34.

17. Kostis WJ, Demissie K, Marcella SW, Shao YH, Wilson AC, Moreyra AE. Weekend versus weekday admission and mortality from myocardial infarction. N Engl J Med. 2007;356(11): 1099-109.

18. McNamara RL, Wang Y, Herrin J, Curtis JP, Bradley EH, Magid DJ, et al. Effect of door-to-balloon time on mortality in patients with ST-segment elevation myocardial infarction. J Am Coll Cardiol. 2006;47(11):2180-6.

19. Roe MT, Messenger JC, Weintraub WS, Cannon CP, Fonarow GC, Dai D, et al. Treatments, trends, and outcomes of acute myocardial infarction and percutaneous coronary intervention. J Am Coll Cardiol. 2010;56(4):254-63.

20. Ortolani P, Marzocchi A, Marrozzini C, Palmerini T, Saia $F$, Aquilina $M$, et al. Clinical comparison of "normalhours" vs "off-hours" percutaneous coronary interventions for ST-elevation myocardial infarction. Am Heart J. 2007; 154(2):366-72.

21. Brodie BR, Gersh BJ, Stuckey T, Witzenbichler B, Guagliumi G, Peruga JZ, et al. When Is Door-to-Balloon Time Critical? Analysis From the HORIZONS-AMI (Harmonizing Outcomes with Revascularization and Stents in Acute Myocardial Infarction) 
Cardoso CO, et al. Angioplastia Primária no Infarto Agudo do Miocárdio: Existe Diferença de Resultados entre as Angioplastias Realizadas Dentro e Fora do Horário de Rotina? Rev Bras Cardiol Invasiva. 2010;18(3):273-80.

and CADILLAC (Controlled Abciximab and Device Investigation to Lower Late Angioplasty Complications) trials. J Am Coll Cardiol. 2010;56(5):407-13.

22. Widimsky P, Wijns W, Fajadet J, de Belder M, Knot J, Aaberge L, et al. Reperfusion therapy for ST elevation acute myocardial infarction in Europe: description of the current situation in 30 countries. Eur Heart J. 2010;31(8): 943-57.

23. Bradley EH, Herrin J, Wang $\mathrm{Y}$, Barton BA, Webster TR, Mattera JA, et al. Strategies for reducing the door-to-balloon time in acute myocardial infarction. N Engl J Med. 2006; 355(22):2308-20. 